\title{
The Canonical Endomorphism for Infinite Index Inclusions
}

\author{
F. Fidaleo and T. Isola
}

\begin{abstract}
We give purely algebraic characterisations of the canonical endomorphism in interesting infinite index cases, continuing previous works of Longo and the authors. We apply these results when compact and discrete (but not necessarily finite-dimensional) Woronowicz algebras act alternately on the factors in the various levels of Jones' tower. We characterise when the acting algebra is a Kac algebra.
\end{abstract}

Keywords: Infinite index inclusions, actions of Kac algebras

AMS subject classification: Primary $46 \mathrm{~L} 37$, secondary $46 \mathrm{~L} 10$

\section{Introduction}

The purpose of this work is to extend some results of [19] beyond the finite index case, namely to find necessary and sufficient algebraic conditions on an endomorphism $\gamma$ of a von Neumann algebra $M$ which guarantee the existence of a subalgebra $N \subset$ $M$ for which $\gamma$ is the associated canonical endomorphism. We solve this problem in the cases (dual to each other) when there is a normal faithful conditional expectation either from $M$ to $N$ or from $N^{\prime}$ to $M^{\prime}$. We use Pimsner-Popa basis (relative to an inclusion possessing a faithful conditional expectation) made of elements of the bigger algebra (and not merely affiliated to it), whose existence we show in case the algebras involved are properly infinite. Finally, we apply our results to the context of Longo's $Q$-systems [19] and give a different characterisation of (semicompact or semidiscrete) depth 2 inclusions, which have been recently proved in $[2,3]$ to be generated as crossed products by Woronowicz algebras [22]. In addition we characterise when the Woronowicz algebra is indeed a Kac algebra, using the formalism of $Q$-systems.

Our main motivation for studying, here and in [5], the canonical endomorphism $\gamma$ of an inclusion $N \subset M$ of von Neumann algebras is that the latter can be interpreted as being generated by means of a crossed product by the action on $N$ of an implicitly defined "quantum object", and $\gamma$ should be regarded in some sense as the "regular representation" of the quantum object. This is to be interpreted by analogy with the case of inclusions $N \subset N \times_{\alpha} G$ coming from outer actions of finite groups, where the

Both authors: Università di Roma "Tor Vergata", Dipartimento di Matematica, Via della Ricerca Scientifica, 00133 Roma, Italy

e-mail: Fidaleo@mat.uniroma2.it

e-mail: Isola@mat.uniroma2.it 
irreducible decomposition of $\lambda$ is $\lambda \cong \oplus_{g \in G} \alpha_{g}$, while $\left.\lambda\right|_{N} \cong \oplus_{\pi \in G} d_{\pi} \rho_{\pi}$, where the $\rho_{\pi}$ are (irreducible) endomorphisms of $N$ in one-to-one correspondence with the irreducible representations $\pi$ of $G$ (see [14], for example).

This paper is organised as follows.

After the preliminary Section 2, Section 3 is devoted to Pimsner-Popa bases. Namely, given an inclusion $N \subset M$ of properly infinite von Neumann algebras with a faithful normal conditional expectation $E: M \rightarrow N$, we construct a Pinsner-Popa basis for the left $N$-module ${ }_{N} M$, completely made of elements of $M$ (not just affiliated to $M$, as it happens in the type $\mathrm{II}_{1}$ case considered by Popa [27]). Using this basis, we characterise in a purely algebraic way when an endomorphism $\lambda \in \operatorname{End}(M)$ is a canonical one. This is done in Section 4 in two cases (dual to each other): first when there exists a faithful conditional expectation $E: N \rightarrow \lambda(M)$, which we call semidiscrete, and secondly when there exists a faithful conditional expectation $E: M \rightarrow N$, which we call semicompact. Section 5 concerns the extension of the notion of Longo's $Q$-system [19] to the semicompact and sernidiscrete cases, and the proof of a duality theory between them. If one implements a $Q$-system concretely as an inclusion of von Neumann algcbras, the dual $Q$-system appears, using the canonical endomorphism, in a natural way in the Jones tunnel

$$
\cdots \subset \gamma(N) \subset \gamma(M) \subset N \subset M .
$$

We conclude with Section 6 where we prove an extension of a Frobenius reciprocity result of [19] and apply it to (semidiscrete and semicompact) $Q$-systems based on a factor-subfactor inclusion of depth 2 , which, as we prove, are characterised as those for which the canonical endomorphism has the "absorbing" property $\lambda^{2} \cong d \lambda$, already known in the case of compact groups [9], and of finite-dimensional Kac algebras [19]. Therefore, using recent results in $[2,3]$ we can show that an irreducible semicompact $Q$-system based on a factor-subfactor inclusion of depth 2 will appear as the crossed product of an irreducible semidiscrete (hence automatically discrete according to the terminology in [11]) $Q$-system by an outer action of a discrete Woronowicz algebra, so that the Jones tunnel is obtained via (alternate) crossed product procedures. The dual case, corresponding to prime actions of compact Woronowicz algebras, leads to a more complicated situation, already well known in case of compact group actions (see [23, 28]). Finally we characterise the case when a Kac algebra appears, improving on [3], namely a discrete Kac algebra, together with its compact dual algebra, appears if and only if the canonical endomorphism $\gamma \in \operatorname{Sect}(M)$ decomposes as

$$
\gamma=\oplus_{i} d\left(\rho_{i}\right) \rho_{i}
$$

where $\left\{\rho_{i}\right\}_{i \in I} \subset \operatorname{Sect}(M)$ is a basis of finite index irreducible sectors for the *-semiring generated by $\left\{\rho_{i}\right\}$ themselves. The above condition is well known for compact (or discrete) groups and finite-dimensional Hopf *-algebras [19].

The cases of arbitrary Woronowicz algebras or more complicated quantum symmetries such as weak and quasi-weak Hopf algebras seem to be very difficult; we hope to return to these open problems in the future. 


\section{Notation and preliminaries}

We consider in the following, for simplicity, only inclusions of von Neumann algebras with separable predual. For the reader's convenience we recall some notation, used throughout the paper.

Let $M \subset \mathcal{B}(\mathcal{H})$ be a von Neumann algebra. Then $s_{M}(e)$ is the support in $M$ of the projection $e \in \mathcal{B}(\mathcal{H})$, and $\operatorname{Proj}(M)$ is the set of projections in $M$. If $N$ is a von Neumann subalgebra of $M$, then $C(M, N)$ and $E(M, N)$ are the set of normal and normal faithful, respectively, conditional expectations from $M$ onto $N$, whereas $P(M, N)$ is the set of normal semifinite faithful $N$-valued weights on $M$. If $T \in P(M, N)$, then

$$
\mathfrak{N}_{T}:=\left\{x \in M: T\left(x^{*} x\right) \in N\right\}
$$

and

$$
\mathfrak{M}_{T}:=\operatorname{span}\left\{x \in M_{+}: T(x) \in N\right\} \equiv \mathfrak{N}_{T}^{*} \mathfrak{N}_{T}
$$

is the domain of $T$. Further,

$$
\left(N^{\prime} \wedge M\right)_{T}:=\left\{x \in N^{\prime} \wedge M: \sigma_{t}^{T}(x)=x \quad(t \in \mathbb{R}\}\right.
$$

is the centralizer of $T . \operatorname{End}(M)$ is the set of normal faithful unital *-endomorphisms of $M$, and $[\rho] \in \operatorname{Sect}(M)$ is the sector determined by $\rho$ in $\operatorname{End}(M)$ modulo inner automorphisms. For $\rho, \sigma \in \operatorname{End}(M),(\sigma, \rho)$ is the vector space of intertwiners between $\rho$ and $\sigma$ :

$$
(\sigma, \rho):=\{v \in M: v \sigma(x)=\rho(x) v(x \in M)\} .
$$

Let $N \subset M$ be an inclusion of properly infinite von Neumann algebras, take a cyclic and separating vector $\Omega$ for both $N$ and $M$ and set $\Gamma:=J_{N}^{\Omega} J_{M}^{\Omega}$, where $J_{M}^{\Omega}$ denotes Tomita's modular conjugation with respect to $(M, \Omega)$, then $\gamma:=\left.\operatorname{ad} \Gamma\right|_{M} \in \operatorname{End}(M)$ is a canonical endomorphism for the inclusion $N \subset M$, and its sector is uniquely determined. Using $\gamma$, a conjugate endomorphism of $\rho \in E n d(M)$ is given by $\bar{\rho}=\rho^{-1} \circ \gamma$. For the general theory of von Neumann algebras we refer the reader to [12, $28-30]$.

Let us now recall the definition of $H$. Kosaki's index [13] based on A. Connes' spatial theory and U. Haagerup's operator-valued weights (see [28]). If $N \subset M$ are von Neumann algebras, associated to every $E \in E(M, N)$ there is an $M^{\prime}$-valued operator weight $E^{-1}$ on $N^{\prime}$ uniquely determined by the rclation

$$
d(\varphi \circ E) / d \psi=d \varphi / d\left(\psi \circ E^{-1}\right)
$$

for all normal semifinite faithful weights $\varphi$ on $N$ and $\psi$ on $M^{\prime}$. Observe that the index of $E, \operatorname{Ind}(E):=E^{-1}(1) \in \overline{Z(M)}_{+}$, the extended positive part of $Z(M)$, does not depend on the representation of $M$ (as the same proof of [13: Theorem 2.2] works).

Let $\varphi$ be a normal faithful state on $N$, and set $\psi:=\varphi \circ E$. Let $\Psi \in \mathcal{H} \equiv \mathcal{H}_{\psi}$ be cyclic and separating for $M$ and such that $\psi=(\cdot \Psi, \Psi)$, and set $e:=[N \Psi] \in N^{\prime}$, the Jones projection of the inclusion. The following propositions summarise standard results on index theory for inclusions. 
Proposition 2.1 (see [13]).

(i) $E^{-1}(e)=1$. $M$ bì $N$.

(ii) $M_{1}:=\langle M, e\rangle \equiv J N^{\prime} J$, where $J \equiv J_{M}^{\Psi}$. This algebra is called the extension of

(iii) If $J$ is a modular conjugation for $M$, and $j:=$ ad $J$, then $E_{1}:=j \circ E^{-1} \circ j \in$ $P\left(M_{1}, M\right) . E_{1}$ is called the dual (operator-valued) weight of $E$.

Proposition 2.2 [5: Theorem 2.1]. Let $N \subset M \subset L$ be von Neumann algebras, $E \in E(M, N)$ and $f \in \operatorname{Proj}(L)$ such that

(i) $f x f=E(x) f \quad(x \in M)$

(ii) $L=\langle M, f\rangle$

(iii) $s_{Z(L)}(f)=1$ and $s_{N}(f)=1$.

Then there is an isomorphism $\phi: L \rightarrow M_{1}$ such that $\left.\phi\right|_{M}=i d_{M}$ and $\phi(f)=e$.

Proposition 2.3. Let $N \subset M$ be properly infinite von Neumann algebras. Then for any $E \in E(M, N)$ there is an isometry $v \in N$ such that $E(x)=v^{*} \gamma(x) v \quad(x \in M)$, $v \in\left(\left.i d\right|_{N},\left.\gamma\right|_{N}\right)$ and $\gamma^{-1}\left(v v^{*}\right)$ is Jones' projection for the inclusion $N \subset M$.

Proof. See [16: Proposition 5.1] or also [5: Lemma 3.3]

For the reader's convenience we report some results in [5] the first of them being based on an argument in [17: Theorem 4.1].

Proposition 2.4. Let $N \subset M$ be properly infinite von Neumann algebras, $\lambda \in$ $\operatorname{End}(M)$, and assume one of the following

(i) there is an isometry $v \in M$ such that $v x=\lambda(x) v(x \in N)$ and $\lambda(M) \subset N \subset$ $\left\langle N, v v^{*}\right\rangle \equiv M$ is the extension of $N$ by $\lambda(M)$

(ii) there is an isometry $v \in N$ such that $v x=\lambda(x) v \quad(x \in \lambda(M))$ and $\lambda(N) \subset$ $\lambda(M) \subset\left\langle\lambda(M), v v^{*}\right\rangle \equiv N$ is the extension of $\lambda(M)$ by $\lambda(N)$.

Then $\lambda$ is a canonical endomorphism for $N \subset M$.

Proof. We just prove (i), as the proof of (ii) is similar. Let $\Omega$ be a cyclic and separating vector for $\lambda(M)$ and $N$, and set $J:=J_{N}^{\Omega}$ and $J_{0}:=J_{\lambda(M)}^{\Omega}$. Set $v_{0}:=J v J \in$ $J\left\langle N, v v^{*}\right\rangle J=\lambda(M)^{\prime}$. Let $\xi$ be cyclic and separating for $N$ such that $v v^{*}=[\lambda(M) \xi]$. The canonical implementation of $y \in \lambda(M) \rightarrow y v v^{*} \in \lambda(M) v v^{*}$ with respect to $\Omega$ and $\xi$ is given by $w_{0}=v_{0} z$, where $z \in \lambda(M)^{\prime}$ is unitary. Then by [16: Proposition 3.1]

$$
\Gamma:=J_{0} J=w_{0}^{*} J w_{0} J=z^{*} v_{0}^{*} J v_{0} J J z J
$$

so that, to compute the sector of $\gamma:=\left.\operatorname{ad} \Gamma\right|_{N}$, we can assume $w_{0}=v_{0}$. Then, for all $x \in N$,

$$
\begin{aligned}
\Gamma x \Gamma^{*} & =v_{0}^{*} J v_{0} J x J v_{0}^{*} J v_{0} \\
& =J v^{*} J v x v^{*} J v J \\
& =J v^{*} J \lambda(x) v v^{*} J v J \\
& =\lambda(x) J v^{*} J v v^{*} J v J \\
& =\lambda(x) J v^{*} v v^{*} v J \\
& =\lambda(x)
\end{aligned}
$$


where we used $J v v^{*} J=v v^{*}$, as $v v^{*}$ is Jones' projection for $\lambda(M) \subset N$. Therefore $\left.\lambda\right|_{N}$ is a canonical endomorphism for $\lambda(M) \subset N$, so that $\lambda$ is a canonical endomorphism for $N \subset M$

Proposition 2.5. Let $N \subset M$ be properly infinite von Neumann algebras, $\rho \in$ End $(M)$ and $v \in(i d, \rho)$ be such that

(i) $\rho(M) \subset N \subset\left\langle N, v v^{*}\right\rangle=: L$ is the extension of $N$ by $\rho(M)$

(ii) $s_{Z(L)}\left(v v^{*}\right)=1$.

Then $M=\left\langle N, v v^{*}\right\rangle$.

Proof. Set $f:=v v^{*}$, and observe that

$$
f\langle N, f\rangle f=f \rho(M) f=\{\rho(x) f: x \in M\}=\left\{v x v^{*}: x \in M\right\}=f M f,
$$

that is $\left\langle N, v v^{*}\right\rangle_{f}=M_{f}$. Therefore $L_{f}^{\prime}=\langle N, f\rangle_{f}^{\prime}=M_{f}^{\prime}$ and, as $s_{Z(L)}(f)=1$, the map $L^{\prime} \rightarrow L_{f}^{\prime}$ is an isomorphism which restricts to the isomorphism $M^{\prime} \rightarrow M_{f}^{\prime} \equiv L_{f}^{\prime}$, so that $L^{\prime}=M^{\prime}$, that is $L=M$

\section{On the Pimsner-Popa basis}

Let $N \subset M$ be an inclusion of von Neumann algebras and $T \in P(M, N)$. A PimsnerPopa basis is a basis for the self-dual completion $X_{T}$ of the left $N$-module ${ }_{N}\left(\mathfrak{M}_{T}\right)$ relative to the $N$-valued inner product $\langle x, y\rangle_{T}:=T\left(x y^{*}\right)$ as in [25]. It is a well-known result that, if $E(M, N) \neq \emptyset$, there exists a Pimsner-Popa basis consisting of clements affiliated to $M$, in the sense explained in [27]. In this section we strengthen this result by showing that, if $N \subset M$ are properly infinite von Neumann algebras, a Pimsner-Popa basis consisting of elements in $M$ can be chosen. The proof also shows how such a basis can be constructed.

We need some preliminary lemmas.

Lemma 3.1. Let $N \subset M$ be an inclusion of properly infinite von Neumann algebras, with $E(M, N) \neq 0$. Then:

(i) Jones' projection $e \in M_{1}$ is properly infinite.

(ii) If $\hat{q} \in \operatorname{Proj}\left(M_{1}\right)$ is majorized by e, then there exists $q \in \operatorname{Proj}(N)$ such that $q e=\hat{q}$.

Proof. (i): We have isometries $v, w \in N$ with orthogonal ranges satisfying $v v^{*}+$ $w w^{*}=1$. We can define $\hat{v}:=v e$ and $\hat{w}:=w e$ and obtain two partial isometries with ortogonal ranges that satisfy $\hat{v}^{*} \hat{v}=e=\hat{w}^{*} \hat{w}$ and $\hat{v} \hat{v}^{*}+\hat{w} \hat{w}^{*}=e$. The assertion follows by [29: Section 4.12].

(ii): As $e M_{1} e \sim N e$ (see [26]), there exists $q \in N$ such that $q e=\hat{q} \equiv e \hat{q} e$. Moreover, $\hat{q} \in \mathfrak{M}_{E_{1}}$ and $q=E_{1}(\hat{q})$ where $E_{1} \in P\left(M_{1}, M\right)$ is the dual weight of $E \in E(M, N)$. Finally, $q \in \operatorname{Proj}(N)$ as $q^{*} q=E_{1}\left(q^{*} q e\right)=E_{1}\left(e q^{*} q e\right)=E_{1}\left(\hat{q}^{*} \hat{q}\right)=E_{1}(\hat{q})=q$

The proof of the following lemma is heavily based on [8: Lemma 2.2]. 
Lemma 3.2. Let $N \subset M$ be an inclusion of von Neumann algebras and $T$ a normal $N$-valued weight on $M$. The following statements are equivalent:

(i) $T$ is semifinite.

(ii) There is $x \in\left(\mathfrak{M}_{T}\right)+$ such that $s_{M}(x)=1$.

(iii) There is a sequence $\left\{p_{n}\right\}$ of orthogonal projections in $\mathfrak{M}_{T}$ such that $\sum p_{n}=1$.

Proof. (i) $\Rightarrow$ (ii): As the unit ball of $M_{+}$is metrizable in the strong topology [30: Proposition 2.7], there is a strongly dense sequence $\left\{x_{n}\right\}$ in the unit ball of $\left(\mathfrak{M}_{T}\right)_{+}$. Then

$$
\sum_{n=1}^{\infty} 2^{-n} \frac{x_{n}}{\left\|1+T\left(x_{n}\right)\right\|}
$$

converges in norm to some $x \in M_{+}$. As $T$ is normal, $x \in\left(\mathfrak{M}_{T}\right)_{+}$. In fact,

$$
T(x)=\sum_{n=1}^{\infty} 2^{-n} \frac{T\left(x_{n}\right)}{\left\|1+T\left(x_{n}\right)\right\|} \in N .
$$

Finally, if $p \in \operatorname{Proj}(M)$ is such that $x p=0$, then

$$
0=\sum_{n=1}^{\infty} 2^{-n} \frac{p x_{n} p}{\left\|1+T\left(x_{n}\right)\right\|}
$$

which implies $p x_{n} p=0$ for all $n$, that is $p x_{n}=0$, which implies $p=0$, due to the density of $\left\{x_{n}\right\}$.

(ii) $\Rightarrow$ (iii): Set $p_{n}:=\chi_{\left(\frac{1}{n+1}, \frac{1}{n}\right)}(x)$. Then $p_{n}$ are mutually orthogonal projections in $M$ and $\sum_{n} p_{n}=s_{M}(x)=1$. Finally, $T\left(p_{n}\right) \leq(n+1) T\left(x p_{n}\right) \leq T(x) \in N_{+}$.

(iii) $\Rightarrow$ (i) is obvious

The following lemma is essentially [11: Lemma 2.2] (which is only proved in the case $N \subset M$ are factors).

Lemma 3.3 (Push-down Lemma). Let $N \subset M$ be an inclusion of von Neumann algebras and $E \in E(M, N)$. Then, for all $x \in \mathfrak{N}_{E_{1}}$, we have $e E_{1}(e x)=e x$.

Proof. The same proof of [11: Lemma 2.2] works. Just observe that $\mathcal{A}=M e M$ is still weakly dense in $M_{1}$. Indeed, as $\overline{\mathcal{A}}$ is a weakly-closed two-sided ideal in $M_{1}$, there is a central projection $z \in M_{1}$ such that $z M_{1}=\overline{\mathcal{A}}$. Then $z=1$ as $(1-z) e=0$, so that $z \geq s_{Z\left(M_{1}\right)}(e)=1$

We can now prove the main result of this section, namely that a basis $\left\{m_{i}\right\}_{i \in I}$ for ${ }_{N} M$ made of elements in $M$ can be chosen.

Remark 3.4. Recall [27] that the following equivalent properties characterise a Pimsner-Popa basis:

(i) $E\left(m_{i} m_{j}^{*}\right)=\delta_{i j} q_{j} \in \operatorname{Proj}(N) \backslash\{0\}$ and $\sum_{i \in I} m_{i}^{*} e m_{i}=1$.

(ii) $m_{i}^{*} e m_{i}$ are mutually ortogonal projections in $M_{1}$, and $\left(\sum_{i} m_{i}^{*} e \mathcal{H}\right)^{-}=\mathcal{H}$. 
In general, the sequence $\left\{m_{i}\right\}$ is made of elements affiliated to $M$, as it is explained in [27].

Theorem 3.5. Let $N \subset M$ be an inclusion of properly infinite von Neumann algebras, and $E \in E(M, N)$. Then there exists a Pimsner-Popa basis for the left module ${ }_{N} M$ made of elements of $M$.

Proof. Let $\left\{p_{i}\right\}_{i \in I}$ be a family of orthogonal projections of $M_{1}$ such that $p_{i} \in \mathfrak{M}_{E_{1}}$ and $\sum_{i \in I} p_{i}=1$, as in Lemma 3.2. Thanks to $s_{Z\left(M_{1}\right)}\left(p_{i}\right) \leq s_{Z\left(M_{1}\right)}(e) \equiv 1$ and $e$ being properly infinite, we can find a collection $\left\{v_{i}\right\}_{i \in l} \subset M_{1}$ of partial isometries such that

$$
v_{i}^{*} v_{i}=p_{i} \quad \text { and } \quad \hat{q}_{i}:=v_{i} v_{i}^{*} \leq e, \quad i \in I
$$

Hence $v_{i} \equiv e v_{i} p_{i} \in \mathfrak{M}_{E_{1}}$ and we take $m_{i}:=E_{1}\left(v_{i}\right)$ as a Pimsner-Popa basis. Indeed, $m_{i}$ is the push-down of $v_{i}$, as $v_{i}=e v_{i}=e E_{1}\left(e v_{i}\right)=e E_{1}\left(v_{i}\right)=e m_{i}$. Furthermore, .

$$
p_{i}=v_{i}^{*} v_{i}=v_{i}^{*} e v_{i}=m_{i}^{*} e m_{i}
$$

and summing up we obtain $\sum_{i} m_{i}^{*} e m_{i}=1$. Moreover, we get

$$
\begin{aligned}
E\left(m_{i} m_{j}^{*}\right) & =E_{1}\left(e E\left(m_{i} m_{j}^{*}\right)\right) \\
& =E_{1}\left(e m_{i} m_{j}^{*} e\right) \\
& =E_{1}\left(e v_{i} v_{j}^{*} e\right) \\
& =\delta_{i j} E_{1}\left(e \hat{q}_{j}\right) \\
& =\delta_{i j} E_{1}\left(e q_{j}\right) \\
& =\delta_{i j} q_{j}
\end{aligned}
$$

and the theorem is proved

Proposition 3.6. Let $N \subset M$ be an inclusion of von Neumann algebras. If $\left\{m_{i}\right\}_{i \in I} \subset M$ is a Pimsner-Popa basis for $N \subset M$, then every $x \in M$ has the expansion

$$
x=\sum_{i \in I} E\left(x m_{i}^{*}\right) m_{i}
$$

where the last sum converges unconditionally relative to the topology generated by the separating family of seminorms $\left\{s_{\varphi}: \varphi \in\left(N_{*}\right)_{+}\right\}$, with $s_{\varphi}(x)=\varphi \circ E\left(x x^{*}\right)^{1 / 2}$.

Proof. Observe that $m_{i}=q_{i} m_{i}$. In fact,

$$
\begin{aligned}
E\left(\left(m_{i}\right.\right. & \left.\left.-q_{i} m_{i}\right)\left(m_{i}-q_{i} m_{i}\right)^{*}\right) \\
& =E\left(m_{i} m_{i}^{*}\right)-E\left(q_{i} m_{i} m_{i}^{*}\right)-E\left(m_{i} m_{i}^{*} q_{i}\right)+E\left(q_{i} m_{i} m_{i}^{*} q_{i}\right) \\
& =0
\end{aligned}
$$

and the claim follows by faithfulness of $E$.

We now prove the convergence of $(1)$ in the topology generated by the above seminorms. As $p_{A}:=\sum_{i \in A} m_{i}^{*} e m_{i} \nearrow 1$ in the strong operator topology when the finite subset $A \subset I$ tends to the whole index set $I$, for $x \in M$ we have

$$
\left\|\left(1-p_{A}\right) \Lambda_{\hat{\varphi}}\left(x^{*} e\right)\right\|_{\hat{\varphi}}^{2} \rightarrow 0, \quad \text { that is } \hat{\varphi}\left(\operatorname{ex}\left(1-p_{A}\right) x^{*} e\right) \rightarrow 0
$$


for every normal semifinite faithful weight $\hat{\varphi}$ on $M_{1}$ given by $\hat{\varphi}:=\varphi \circ E \circ E_{1}\left(\varphi \in\left(N_{*}\right)_{+}\right)$. Finally, we get

$$
\begin{aligned}
\hat{\varphi}\left(e x\left(1-p_{A}\right) x^{*} e\right) & =\varphi \circ E\left(x x^{*}\right)-\sum_{i \in A} \varphi \circ E\left(E\left(x m_{i}^{*}\right) E\left(m_{i} x^{*}\right)\right) \\
& =\varphi \circ E\left(x x^{*}\right)-\sum_{i, j \in A} \varphi \circ E\left(E\left(x m_{i}^{*}\right) m_{i} m_{j}^{*} E\left(m_{j} x^{*}\right)\right) \\
& \equiv \varphi \circ E\left(\left(x-x_{A}\right)\left(x^{*}-x_{A}^{*}\right)\right)
\end{aligned}
$$

where $x_{A}:=\sum_{i \in A} E\left(x m_{i}^{*}\right) m_{i}$ and we have applied $m_{i}=q_{i} m_{i}$ and $E\left(m_{i} m_{j}^{*}\right)=\delta_{i j} q_{j}$

Remark 3.7. Note that

$$
\operatorname{Ind} E=\sum_{i \in I} m_{i}^{*} m_{i} \in{\overline{Z(M)_{+}}}_{+}
$$

and the index is finite if and only if $\sum_{i \in I} m_{i}^{*} m_{i} \in Z(M)$.

As a direct consequence of Theorem 3.5 we have the following

Corollary 3.8. The self-dual completion ${ }_{N} X$ of the left $N$-module ${ }_{N} M$ is isomorphic to the ultraweak direct sum

$$
{ }_{N} X \cong \bigoplus_{j \in J} N q_{j}
$$

Proof. It follows by the above considerations and [25: Theorem 3.12]

\section{The canonical endomorphism in the semidiscrete and semicompact cases}

In this section we provide the announced conditions which are equivalent to the fact that an endomorphism is canonical. We treat only the semidiscrete and semicompact cases, that is the cases when the canonical endomorphism $\lambda$ is associated to an inclusion $N \subset M$ such that $E(N, \lambda(M)) \neq \emptyset$ or $E(M, N) \neq \emptyset$. These conditions allow us to extend the definition of a $Q$-system given in [19] to non-trivial examples of infinite index.

Theorem 4.1 (Semidiscrete case). Let $M$ be a properly infinite von Neumann algebra and $\lambda \in \operatorname{End}(M)$. Then the following statements are equivalent:

(i) There is a von Neumann subalgebra $N$ of $M$, with $E(N, \lambda(M)) \neq \emptyset$, such that $\lambda$ is a canonical endomorphism for $N \subset M$.

(ii) There are an isometry $v \in(i d, \lambda)$ and $\left\{m_{i}\right\} \subset M$ such that $\sum_{i} m_{i}^{*} v v^{*} m_{i}=1$ and, setting $N:=\left\langle\lambda(M),\left\{m_{i}\right\}\right\rangle$, one has that $x v=0$ for $x \in N$ implies $x=0$.

Moreover, if $v^{*} m_{i} m_{j}^{*} v=\delta_{i j} q_{i}$ with $q_{i} \in \operatorname{Proj}(\lambda(M)) \backslash\{0\}$, then $\left\{m_{i}\right\}$ is a PimsnerPopa basis for the inclusion $\lambda(M) \subset N$.

Proof. (i) $\Rightarrow$ (ii): Let $E \in E(N, \lambda(M))$. Then from Proposition 2.3 it follows that there is an isometry $v \in M$ such that $\lambda(x) v=v x \quad(x \in M), \lambda\left(v^{*} \cdot v\right)=E$ and, 
finally, $v v^{*}$ is Jones' projection for the inclusion $\lambda(M) \subset N$. By Theorem 3.5 there are $\left\{m_{i}\right\}_{i \in I} \subset N$ such that

$$
\sum_{i \in I} m_{i}^{*} v v^{*} m_{i}=1 \quad \text { and } \quad N=\left\langle\lambda(M),\left\{m_{i}\right\}\right\rangle
$$

which can be shown as in the proof of [6: Proposition 6]. Finally, if $x \in N$ is such that $x v=0$, then $0=\lambda\left(v^{*} x^{*} x v\right)=E\left(x^{*} x\right)$, which implies $x=0$, as $E$ is faithful.

(ii) $\Rightarrow$ (i): Let us set $E:=\lambda\left(v^{*} \cdot v\right) \in C(N, \lambda(M))$. Then $E$ is faithful as $E(p)=0$ for some $p \in \operatorname{Proj}(N)$ implies $p v=0$, and therefore $p=0$. We want to prove that $\lambda(M) \subset N \subset\left\langle N, v v^{*}\right\rangle$ is the extension of $N$ by $\lambda(M)$. Indeed, setting $f:=v v^{*}$, we observe that $E(x) f=f x f(x \in N)$ so that $s_{\lambda(M)}(f)=1$, from [5: Lemma 3.2/(ii)]. Finally,

$$
s_{Z\left(\left\langle N, v v^{\bullet}\right\rangle\right)}(f) \geq s_{N^{\prime} \wedge M}(f)=1
$$

because, if $p \in \operatorname{Proj}\left(N^{\prime} \wedge M\right)$ is such that $p v v^{*}=0$, then

$$
p=p \sum_{i} m_{i}^{*} v v^{*} m_{i}=\sum_{i} m_{i}^{*} p v v^{*} m_{i}=0
$$

From Proposition 2.2 we conclude.

Therefore, by Proposition 2.5, $\left\langle N, v v^{*}\right\rangle \equiv M$ and, by Proposition $2.4, \lambda$ is a canonical endomorphism for $N \subset M$

Theorem 4.2 (Semicompact case). Let $M$ be a properly infinite von Neumann algebra and $\lambda \in \operatorname{End}(M)$. Then the following statements are equivalent:

(i) There is a von Neumann subalgebra $N$ of $M$ with $E(M, N) \neq \emptyset$ such that $\lambda$ is a canonical endomorphism for $N \subset M$.

(ii) There are an isometry $v \in\left(\lambda, \lambda^{2}\right)$ and $\left\{m_{i}\right\} \subset \lambda(M)$ such that $\sum_{i} m_{i}^{*} v v^{*} m_{i}=$ 1, $\lambda(v) v=v^{2}$ and $\lambda(v)^{*} v=v v^{*}$, and such that $\lambda(x) v=0$ for $x \in M$ implies $x=0$.

Moreover, if $v^{*} m_{i} m_{j}^{*} v=\delta_{i j} q_{i}$ for $q_{i} \in \operatorname{Proj}(N) \backslash\{0\}$, then $\left\{m_{i}\right\}$ is a Pimsner-Popa basis for the inclusion $N \subset M$.

Proof. (i) $\Rightarrow$ (ii): Let $E \in E(M, N)$. Then from Proposition 2.3 it follows that there is an isometry $v \in N$ such that $\lambda(x) v=v x(x \in N), v^{*} \lambda(\cdot) v=E$ and, finally, $v v^{*}$ is Jones' projection for the inclusion $\lambda(N) \subset \lambda(M)$. Therefore $\lambda(\lambda(x)) v=v \lambda(x) \quad(x \in$ $M)$, that is $v \in\left(\lambda, \lambda^{2}\right)$. Further, $\lambda(v) v=v^{2}$ and $\lambda(v)^{*} v=v v^{*}$ are immediate. Besides, if $x \in M$ and $\lambda(x) v=0$, then $\lambda(x) v v^{*}=0$ so that $\lambda(x)=0$ and $x=0$ as $v v^{*}$ is separating for $\lambda(M)$. Finally, by Theorem 3.5 there are $\left\{m_{i}\right\} \subset \lambda(M)$ such that $\sum_{i} m_{i}^{*} v v^{*} m_{i}=1$.

(ii) $\Rightarrow$ (i): Let us set $E:=v^{*} \lambda(\cdot) v$. Then, as in [19: Proposition 5.2], $E \in C(M, N)$ where $N:=E(M)$ is a von Neumann subalgebra of $M$. We want to show that $E$ is faithful. So let $x \in M$ be such that $E\left(x^{*} x\right)=0$. Then $v^{*} \lambda\left(x^{*} x\right) v=0$, that is $\lambda(x) v=0$ and $x=0$, from the hypothesis.

Let us observe that $f:=v v^{*} \in N$, as

$$
E\left(v v^{*}\right)=v^{*} \lambda(v) \lambda(v)^{*} v=v v^{*} v v^{*}=v v^{*}
$$


We want to prove that

$$
\lambda(N) \subset \lambda(M) \subset\langle\lambda(M), f\rangle
$$

is the extension of $\lambda(M)$ by $\lambda(N)$, by using Proposition 2.2. At first, $s_{\lambda(M)}(f)=1$ because, if $\lambda(p) \in \operatorname{Proj}(\lambda(M))$ is such that $\lambda(p) v v^{*}=0$, then $p=0$. Besides

$$
s_{Z(\langle\lambda(M), f\rangle)}(f) \geq s_{\lambda(M)^{\prime} \wedge N}(f)=1
$$

because if $p \in \operatorname{Proj}\left(\lambda(M)^{\prime} \wedge N\right)$ is such that $p v v^{*}=0$, then

$$
p=p \sum_{i} m_{i}^{*} v v^{*} m_{i}=\sum_{i} m_{i}^{*} p v v^{*} m_{i}=0 .
$$

Finally, observing that

$$
F:=\lambda \circ E \circ \lambda^{-1} \in E(\lambda(M), \lambda(N))
$$

is such that $F(x) f=f x f(x \in \lambda(M))$, we get the claim. Therefore, by Proposition 2.5, $\langle\lambda(M), f\rangle \equiv N$ and, by Proposition 2.4, $\lambda$ is a canonical endomorphism for $N \subset M$

Remark 4.3. The above properties are also equivalent to the following one:

(iii) There exists an isometry $v \in\left(\lambda, \lambda^{2}\right)$ such that $\lambda(v) v=v^{2}, \lambda(v)^{*} v=v v^{*}$ and

$$
s_{\lambda(M)^{\prime} \wedge M}\left(v v^{*}\right)=s_{\lambda\left(\lambda(M)^{\prime} \wedge M\right)}\left(v v^{*}\right)=1
$$

as follows from [5: Proposition 6.1].

In the following sections we extend the notion of $Q$-system to semidiscrete and semicompact inclusions and analyse the Takesaki duality which naturally appears considering the Jones tunnel, and the canonical mirroring on it. As an application we have conditions on the canonical endomorphism which are equivalent to the fact that an inclusion arises as a crossed product by a compact or discrete Woronowicz (or Kac) algebra. In the discrete case these conditions can be stated directly in terms of $Q$-systems.

\section{Duality for $Q$-systems}

$Q$-systems in the finite index case were introduced in [19] to consider the canonical endomorphism as a relevant mean to handle the problem of the actions of "quantum symmetries" on von Neumann algebras. This situation typically appears in low-dimensional Quantum Field Theory. Indeed, in the physical Minkowski space, an ordinary compact group acts on the algebra of fields, but, for low-dimensional theories, a braid group statistics appears, so a quantum symmetry is expected to act on the field algebra [20, 21].

We analyse $Q$-systems in the (semidiscrete and semicompact) infinite index case and apply the results to depth 2 factor-subfactor inclusions, that is when compact or discrete Woronowicz algebras naturally appear [2]. This section extends [19: Section 6].

Although some of the properties required in the following definition are unnecessary to characterise a canonical endomorphism $\lambda$ we prefer to define a $Q$-system such that the sequence $\left\{m_{i}\right\}$ directly provides a Pimsner-Popa basis for the relevant inclusions. 


\section{Definition 5.1.}

(i) A $Q$-system of semidiscrete type (semidiscrete $Q$-system for short) is a couple $(M, \Lambda)$ where $M$ is a properly infinite von Neumann algebra and $\Lambda:=\left(\lambda, v,\left\{m_{i}\right\}_{i \in I}\right)$ satisfies the properties given in Theorem 4.1. Namely, $v \in(i d, \lambda)$ is an isometry and $\left\{m_{i}\right\} \subset M$ are such that

$$
\sum_{i} m_{i}^{*} v v^{*} m_{i}=1 \quad \text { and } \quad v^{*} m_{i} m_{j}^{*} v=\delta_{i j} q_{i}
$$

with $q_{i} \in \operatorname{Proj}(\lambda(M)) \backslash\{0\}$, and, setting $N:=\left\langle\lambda(M),\left\{m_{i}\right\}\right\rangle$, one has that $x v=0$ for $x \in N$ implies $x=0$.

(ii) A $Q$-system of semicompact type (semicompact $Q$-system for short) is a couple $(M, \Lambda)$ where $M$ is a properly infinite von Neumann algebra and $\Lambda:=\left(\lambda, v,\left\{m_{i}\right\}_{i \in I}\right)$ satisfies the properties given in Theorem 4.2. Namely, $v \in\left(\lambda, \lambda^{2}\right)$ is an isometry such that $\lambda(v) v=v^{2}, \lambda(v)^{*} v=v v^{*}$, and $\left\{m_{i}\right\} \subset \lambda(M)$ are such that

$$
\sum_{i} m_{i}^{*} v v^{*} m_{i}=1 \quad \text { and } \quad v^{*} m_{i} m_{j}^{*} v=\delta_{i j} q_{i}
$$

with $q_{i} \in \operatorname{Proj}(N) \backslash\{0\}$, and $\lambda(x) v=0$ for $x \in M$ implies $x=0$.

As we have proved, $\lambda$ is the canonical endomorphism for an inclusion of von Neumann algebras $N \subset M$. Contrary to what we did in [5], but in accordance with [11] we say that a properly infinite subalgebra $N$ of a von Neumann algebra $M$ is

semicompact if $E(M, N) \neq \emptyset$

semidiscrete if $E\left(N^{\prime}, M^{\prime}\right) \neq \emptyset$

the last being equivalent to $E\left(M_{1}, M\right) \neq \emptyset$ and to $E(N, \gamma(M)) \neq \emptyset$, with $\gamma$ a canonical endomorphism for $N \subset M$. Therefore, if we say that an inclusion $N \subset M$ is a concrete $Q$-system, the two previous definitions coincide.

We now deal with the irreducibility property of an inclusion $N \subset M$, which seems to be related to the structure of $\left(i d_{N},\left.\gamma\right|_{N}\right)$, where $\gamma$ is the canonical endomorphism of the inclusion (see $[5,16])$. The following result partially confirms the above considerations.

Theorem 5.2. Let $A \subset B$ be a semicompact inclusion of properly infinite von Neumann algebras. The following statements are equivalent:

(i) $A \subset B$ is irreducible, that is $A^{\prime} \wedge B=Z(A)$.

(ii) $E(B, A)$ is a singleton.

(iii) The $Z(A)$-module $\left(i_{A},\left.\gamma\right|_{A}\right)$ is cyclic.

Proof. (i) $\Leftrightarrow$ (ii) is a well-known result by Combes and Delaroche [1].

(iii) $\Rightarrow$ (ii): Let $v_{0}$ be a generator of the $Z(A)$-module $\left(i d_{A},\left.\gamma\right|_{A}\right)$, which must be a (multiple of an) isometry as follows from Proposition 2.3. Therefore, again by the same Proposition 2.3, $E(B, A)$ must be a singleton.

(ii) $\Rightarrow$ (iii): Let $s \in\left(i d_{A},\left.\gamma\right|_{A}\right)$. Then $s^{*} s \in\left(i d_{A}, i d_{A}\right) \equiv Z(A)$ and we can restrict ourselves to the case when $s=v=v p$, where $v \in\left(i d_{A},\left.\gamma\right|_{A}\right)$ is a partial isometry with $p \in Z(A)$ as domain projection and a subprojection of $p$ as range projection. As by Proposition 2.3 there is an isometry $v_{0} \in\left(i d_{A},\left.\gamma\right|_{A}\right)$, we can construct $w:=v p+v_{0} p^{\perp}$ which is an isometry. By the following Lemma 5.3 there is a $z \in Z(A)$ such that $w=z v_{0}$, so that $s=v=v p=z p v_{0}$, that is $v_{0}$ is a generator 
Lemma 5.3. Let $A \subset B$ be properly infinite von Neumann algebras and assume that $E(B, A)$ is a singleton. If $v, w \in\left(i d_{A},\left.\gamma\right|_{A}\right)$ are isometries, then there is a unitary $z \in Z(A)$ such that $v=z w$.

Proof. As $v^{*} \gamma(\cdot) v, w^{*} \gamma(\cdot) w \in C(B, A) \equiv E(B, A)$, because of the hypothesis, we get $E:=v^{*} \gamma(\cdot) v=w^{*} \gamma(\cdot) w$. Let us prove that $e:=v v^{*}$ and $f:=w w^{*}$ are Jones' projections for the inclusion $\gamma(A) \subset \gamma(B)$. Indeed, setting

$$
F:=\gamma \circ E \circ \gamma^{-1} \in E(\gamma(B), \gamma(A))
$$

we have $F(x) e=$ exe $(x \in \gamma(B))$ so that $s_{\gamma(A)}(e)=1$, and

$$
s_{Z(\langle\gamma(B), e\rangle)}(e) \geq s_{\gamma(B)^{\prime} \wedge A}(e)=s_{Z(A)}(e)=1
$$

because, if $p \in \operatorname{Proj}(Z(A))$ is such that $p e=0$, then $p=0$, therefore from Proposition 2.2 we conclude. Then $\langle\gamma(B), e\rangle=A$ as follows from Proposition 2.5. Analogously, $f$ is Jones' projections for the inclusion $\gamma(A) \subset \gamma(B)$ and $\langle\gamma(B), f\rangle=A$. Therefore, by $\mid 14$ : Appendix $\mathrm{A}$ ], $f=e$. Let us now consider

$$
v w^{*}=e v w^{*} e \in e A e=\gamma(A) e,
$$

that is, there exists $z \in A$ such that

$$
v w^{*}=\gamma(z) e=\gamma(z) w w^{*}
$$

which implies $v=\gamma(z) w=w z$ and $w^{*} v=z$. But we have also $w^{*} v \in Z(A)$, so that $1=v^{*} w z=z^{*} z$, and $z$ is a unitary operator in $Z(A)$

The above theorem suggests the following

\section{Definition 5.4.}

(i) A $Q$-system of semidiscrete type $(M, \Lambda)$ is called irreducible if there is a unique $v \in(i d, \lambda)$, up to multiplication by a unitary operator in $Z(M)$.

(ii) A $Q$-system of semicompact type $(M, \Lambda)$ is called irreducible if there is a unique $v \in\left(i d_{N},\left.\lambda\right|_{N}\right)$, up to multiplication by a unitary operator in $Z(N)$.

Hence in both cases the irreducibility condition on the $Q$-system means that a suitable inclusion in the Jones tower

$$
\cdots \subset \gamma(M) \subset N \subset M \subset M_{1} \subset M_{2} \subset \cdots
$$

is irreducible.

There is a natural notion of isomorphism between $Q$-systems, namely $\left(M_{1}, \Lambda_{1}\right)$ and $\left(M_{2}, \Lambda_{2}\right)$ are isomorphic if there is an isomorphism $\varphi: M_{1} \rightarrow M_{2}$ such that $\lambda_{2}=$ $\varphi \circ \lambda_{1} \circ \varphi^{-1}, v_{2}=\varphi\left(v_{1}\right)$ and $m_{2 i}=\varphi\left(m_{1 i}\right)$. It is easy to see that a $Q$-system isomorphic to a semidiscrete one, is itself semidiscrete. Analogously for the semicompact case. 


\section{Definition 5.5.}

(i) $Q$-systems of discrete type $\left(M, \Lambda_{1}\right)$ and $\left(M, \Lambda_{2}\right)$ are inner conjugate if there is a unitary operator $u \in M$ such that $\lambda_{2}=u \lambda_{1}(\cdot) u^{*}, v_{2}=u v_{1}$ and $m_{2 i}=u m_{1 i}$.

(ii) $Q$-systems of compact type $\left(M, \Lambda_{1}\right)$ and $\left(M, \Lambda_{2}\right)$ are inner conjugate if there is a unitary operator $u \in M$ such that $\lambda_{2}=u \lambda_{1}(\cdot) u^{*}, v_{2}=u \lambda_{1}(u) v_{1} u^{*}$ and $m_{2 i}=$ $u \lambda_{1}(u) m_{1 i} u^{*}$.

Finally, $Q$-systems are cocycle equivalent if the first is isomorphic to an inner conjugate copy of the second.

Theorem 5.6 (cfr. [19: Theorem 6.1].) Let $M$ be a properly infinite von Neumann algebra. Then there is a bijective correspondence between (irreducible) semidiscrete subalgebras $N$ of $M$ and (irreducible) semidiscrete $Q$ systems based on $M$. Conjugate inclusions correspond to cocycle equivalent $Q$-systems. The same statement holds if we replace semidiscrete with semicompact everywhere.

Proof. Bijective correspondence follows from Theorems 4.1 and 4.2. Irreducibility assumption for a $Q$-system corresponds to irreducibility of the subalgebra by Theorem 5.2. Finally, cocycle equivalence follows from the Radon-Nikodym property of the canonical endomorphism [15]

Even though it is not needed in the sequel, we now describe, for the sake of completeness, Takesaki duality in the context of $Q$-systems.

Let $\left(M, \lambda, v,\left\{m_{i}\right\}\right)$ be a semidiscrete $Q$-system and $N \subset M$ the corresponding subalgebra. Then $\lambda$ is a canonical endomorphism for $N \subset M$, that is $\lambda=a d \Gamma$, with $\Gamma=J_{N} J_{M}$.

Definition 5.7. $\left(\tilde{M}, \tilde{\lambda}, \tilde{v},\left\{\tilde{m}_{i}\right\}\right)$ is called the dual $Q$-system, where $\tilde{M}:=\Gamma^{*} N \Gamma$ is the crossed product of $M$ by $\Lambda, \tilde{\lambda}:=\left.a d \Gamma\right|_{\bar{M}}, \tilde{v}:=v$ and $\tilde{m}_{i}:=m_{i}$.

Let $\left(M, \lambda, v,\left\{m_{i}\right\}\right)$ be a semicompact $Q$-system and $N \subset M$ the corresponding subalgebra. Then $\lambda$ is a canonical endomorphism for $N \subset M$, that is $\lambda=a d \Gamma$, with $\Gamma=J_{N} J_{M}$.

Definition 5.8. $\left(\tilde{M}, \tilde{\lambda}, \tilde{v},\left\{\tilde{m}_{i}\right\}\right)$ is called the dual $Q$-system, where $\tilde{M}:=\Gamma^{*} N \Gamma$ is the crossed product of $M$ by $\Lambda, \tilde{\lambda}:=\left.a d \Gamma\right|_{\tilde{M}}, \tilde{v}:=\Gamma^{*} v \Gamma$ and $\tilde{m}_{i}:=\Gamma^{*} m_{i} \Gamma$.

It is easy to see that the dual of a semidiscrete $Q$-system is semicompact and viceversa.

In this context Takesaki duality holds, too. The bidual $Q$-system, that is the double crossed product, is obtained by shifting all the structure two steps upwards in Jones' tower. 


\section{Actions of Woronowicz and Kac algebras}

The problem of understanding the structure of depth 2 irreducible inclusions of infinite factors has been recently considered by some authors $[2,3,19]$. In [19] only finite index inclusions are dealt with, whereas in $[2,3]$ the general case is studied, and depth 2 inclusions are characterised as being generated by a crossed product by means of a Woronowicz algebra. Some concrete computations in the semicompact case are also contained in [24] in view of possible applications in Physics. In this section we give a different characterisation of depth 2 irreducible (semicompact or semidiscrete) inclusions of infinite factors, and determine when the acting Woronowicz algebra is a Kac algebra.

The following proposition, while being the crucial step in proving Theorem 6.2, could be considered as a version of Frobenius reciprocity in its own right.

Proposition 6.1. Let $\rho(M) \subset M$ be an irreducible endomorphic inclusion of infinite factors and $\sigma \in \operatorname{End}(M)$.

(i) Suppose that $\sigma \rho \succ i d$. Then $\sigma \succ \bar{\rho}$.

(ii) Suppose that $\rho \sigma \succ i d$. Then $\sigma \succ \bar{\rho}$.

Proof. (i): Let $v \in(i d, \sigma \rho)$, and let $u \in M$ bc an isometry such that

$$
u u^{*}=p:=s_{\sigma(M)^{\prime} \wedge M}\left(v v^{*}\right) \leq 1
$$

(as $p$ is an infinite projection), so that $u u^{*} v=v$, and set $\tau:=u^{*} \sigma(\cdot) u$. Then $\tau \in$ $\operatorname{End}(M)$, as it is easily shown. Set $z:=u^{*} v$, which is an isometry and satisfies

$$
\tau \rho(x) z=u^{*} \sigma \rho(x) u u^{*} v=u^{*} \sigma \rho(x) v=u^{*} v x=z x
$$

so that $z \in(i d, \tau \rho)$, that is $\tau \rho \succ i d$. Besides $s_{\tau \rho(M)^{\prime} \wedge r(M)}\left(z z^{*}\right)=1$, as $\rho(M)^{\prime} \wedge M=\mathbb{C}$.

If we could prove that $s_{\tau(M) \wedge} \wedge\left(z z^{*}\right)=1$, by Proposition 7.1 we would have $\sigma \succ$ $\tau \cong \bar{\rho}$. So all that is left to prove is $s_{\tau(M)^{\prime} \wedge M}\left(z z^{*}\right)=1$.

First let us prove that $(\tau, \tau)=u^{*}(\sigma, \sigma) u$. Indeed, $a \in(\tau, \tau)$ is equivalent to $a u^{*} \sigma(x) u=u^{*} \sigma(x) u a(x \in M)$, which implies

$$
u a u^{*} \sigma(x) u u^{*}=u u^{*} \sigma(x) u a u^{*}, \quad \text { that is } u a u^{*} \sigma(x)=\sigma(x) u a u^{*}
$$

as $u u^{*} \in(\sigma, \sigma)$. Setting $b:=u a u^{*}$, we get $a=u^{*} b u$, with $b \in(\sigma, \sigma)$. Conversely, if $b \in(\sigma, \sigma)$, we get

$$
u^{*} b u \tau(x)=u^{*} b u u^{*} \sigma(x) u=u^{*} \sigma(x) b u=u^{*} \sigma(x) u u^{*} b u=\tau(x) u^{*} b u .
$$

Besides, if $q \in(\tau, \tau)$ is a projection such that $q z z^{*}=0$, then $q=u^{*} f u$, with $f \in(\sigma, \sigma)$. As $q=q^{*} q$ implies $u^{*} f u=u^{*} f^{*} u u^{*} f u$, we can, substituting $f^{*} u u^{*} f$ for $f$, consider $f$ positive. Then $q z z^{*}=0$ if and only if $q z=0$, that is $u^{*} f u u^{*} v=0$. This implies $v^{*} u u^{*} f u u^{*} v=v^{*} f v=0$, which is equivalent to $f v v^{*}=0$, that is $s(f) v v^{*}=0$. Recalling $u u^{*}=s_{(\sigma, \sigma)}\left(v v^{*}\right)$, this implies $s(f) u u^{*}=0$, that is fuu* $=0$, and finally $q=0$.

(ii): The proof is the same as above if one looks at $v \in(i d, \rho \sigma)$ and takes $p:=$ $\rho^{-1}\left(s_{\rho\left(\sigma(M)^{\prime} \wedge M\right)}\left(v v^{*}\right)\right)$ which is an infinite projection. Hence there exists an isometry $u$ such that $u u^{*}=p$. In this case $\tau:=u^{*} \sigma(\cdot) u$ gives rise to a conjugate of $\rho$ with the isometry $\rho\left(u^{*}\right) v$ which intertwines $i d$ and $\rho \tau$ 
We are now ready to extend Frobenius reciprocity (in Longo's setting [19]) to the semidiscrete and semicompact cases.

Theorem 6.2. Let $M$ be an infinite factor and $\rho, \eta \in \operatorname{Sect}(M)$ be irreducible sectors.

(i) (Semidiscrete case): Suppose that $\rho \bar{\rho} \succ i d, \eta \bar{\eta} \succ i d$ and $\alpha \in \operatorname{Sect}(M)$ is a sum of finite index sectors. Then for every $\beta \in \operatorname{Sect}(M)$ we have

$$
\alpha \rho \beta \succ \eta \quad \Longleftrightarrow \quad \bar{\alpha} \eta \bar{\beta} \succ \rho
$$

with equal multiplicities.

(ii) (Semicompact case): Suppose that $\bar{\rho} \rho \succ i d, \bar{\eta} \eta \succ i d$ and $\beta \in \operatorname{Sect}(M)$ is a sum of finite index sectors. Then for every $\alpha \in \operatorname{Sect}(M)$ we have

$$
\alpha \rho \beta \succ \eta \quad \Longleftrightarrow \quad \bar{\alpha} \eta \bar{\beta} \succ \rho
$$

with equal multiplicities.

Proof. It. is the same as in [19], making repeated use of Proposition 6.1

Now we can apply the previous results to the duality for semidiscrete (or equally well semicompact) factor-subfactor inclusions of depth 2, that is when discrete and compact dual Woronowicz algebras (see [22]) alternately act on Jones' tower [2,3]. Moreover, we provide a condition on the canonical endomorphism for the above inclusion to be generated by the crossed product by a (discrete or compact) Kac algebra.

Theorem 6.3. Let $N \subset M$ be an irreducible inclusion of infinite factors.

(a) Suppose that $\gamma$ contains the identity sector. Then the following statements are equivalent:

(i) $N \subset M$ is depth 2 .

(ii) $\gamma^{2} \cong d \cdot \gamma$ for some $d \in \mathbb{N} \cup\{\infty\}$.

(iii) $M$ is the crossed product of $N$ by an outer action of a compact Woronowicz algebra.

(b) Suppose that $\left.\gamma\right|_{N}$ contains the identity sector. Then the following statements are equivalent:

(i) $N \subset M$ is depth 2 .

(ii) $\gamma^{2} \cong d \cdot \gamma$ for some $d \in \mathbb{N} \cup\{\infty\}$.

(iii) $M$ is the crossed product of $N$ by an outer action of a discrete Woronowicz algebra.

Proof. (i) $\Longleftrightarrow$ (ii): It is a consequence of the following Proposition 6.4.

(i) $\Longleftrightarrow$ (iii): It is contained in $[2,3]$

Proposition 6.4. Let $N \subset M$ be an inclusion of infinite factors and $\gamma: M \rightarrow N$ the canonical endomorphism. Suppose that $\gamma$ contains the identity sector and consider the following statements:

(i) $N \subset M$ is depth 2. 
(ii) $\gamma^{2} \cong d \cdot \gamma$ for some $d \in \mathbb{N} \cup\{\infty\}$.

(iii) There exists a sequence of finite index irreducible sectors $\left\{\rho_{i}\right\}_{i \in I} \subset \operatorname{Sect}(M)$ which is a basis for the -semiring generated by the $\left\{\rho_{i}\right\}$ themselves, such that $\gamma=$ $\oplus_{i} d\left(\rho_{i}\right) \rho_{i}$.
Then
(i) $\Longleftrightarrow$
(ii) $\Leftarrow$ (iii)

Proof. Up to tensoring with an absorbing factor [18], we may assume that $N=$ $\rho(M)$ for some irreducible endomorphism $\rho \in \operatorname{End}(M)$. We have $v \in(i d, \rho \bar{\rho}) \subset(\rho, \rho \bar{\rho} \rho)$ and $\bar{\rho}(v) \in(\bar{\rho}, \bar{\rho} \rho \bar{\rho})$, hence the inclusion is regular according to [2: Section 5 ].

By [2: Proposition 6.3], condition (i) means that $\rho \bar{\rho} \rho \cong d \cdot \rho$, whereas condition (ii) translates into $\rho \bar{\rho} \rho \bar{\rho} \cong d \cdot \rho \bar{\rho}$.

(i) $\Rightarrow$ (ii): This follows by multiplying the relation $\rho \bar{\rho} \rho \cong d \cdot \rho$ on the right by $\bar{\rho}$.

(ii) $\Rightarrow$ (i): Note that if $d \in \mathbb{N}$, the proof is contained in [19: Lemma 6.3]. In the general case we may proceed as follows.

Let us set $\sigma:=\rho \bar{\rho} \rho$. Then $\sigma \bar{\rho} \cong d \rho \bar{\rho} \succ d \cdot i d \succ i d$. Therefore, by a repeated application of Proposition 6.1, we obtain $\sigma \cong k \rho \oplus \tau$, where $k \in \mathbb{N} \cup\{\infty\}$ and $\tau \nsucc \rho$. We want to prove that $k=d$ and $\tau=0$.

Suppose that $\tau \neq 0$. Then, as $\rho \bar{\rho} \rho \cong k \rho \oplus \tau$, we have $d \rho \bar{\rho} \cong \rho \bar{\rho} \rho \bar{\rho} \cong k \rho \bar{\rho} \oplus \tau \bar{\rho}$. Therefore $\tau \bar{\rho} \succ \rho \bar{\rho}$. Then $\tau \bar{\rho} \succ i d$ and, by Proposition 6.1 applied to $\bar{\rho}, \tau \succ \rho$ which is absurd. Then $\tau=0$, so that $k=d$.

(iii) $\Rightarrow$ (ii): Due to completeness we have

$$
\rho_{i} \rho_{j}=\oplus_{k} N_{i, j}^{k} \rho_{k} \quad \text { with } \quad \sum_{k} N_{i, j}^{k} d_{k}=d_{i} d_{j}
$$

with finite sum, because of finite index condition. It follows from Frobenius reciprocity that

$$
N_{i, j}^{k} \rho_{k} \prec \rho_{i} \rho_{j} \quad \Longleftrightarrow \quad N_{i, j}^{k} \rho_{i} \prec \rho_{k} \bar{\rho}_{j}
$$

Again by completeness we get

$$
\rho_{k} \bar{\rho}_{j}=\oplus_{k} N_{k, l(j)}^{i} \rho_{i}
$$

where $j \rightarrow l(j)$ is the permutation relative to the conjugation, hence we have $N_{i, j}^{k}=$ $N_{k, l(j)}^{i}$. Finally we have

$$
\begin{aligned}
\gamma^{2} & =\bigoplus_{i j k} d_{i} d_{j} N_{i, j}^{k} \rho_{k} \\
& =\bigoplus_{i j k} d_{i} d_{j} N_{k, l(j)}^{i} \rho_{k} \\
& =\bigoplus_{k} \sum_{i j} d_{i} d_{j} N_{k, l(j)}^{i} \rho_{k} \\
& =\bigoplus_{k} \sum_{j} d_{j} d_{k} d_{l(j)} \rho_{k} \\
& =\bigoplus_{k} d_{k}\left(\sum_{j} d_{j}^{2}\right) \rho_{k} \\
& =d \cdot \gamma
\end{aligned}
$$

where $d \equiv \sum_{j} d\left(\rho_{j}\right)^{2}$ 
We apply this result to $Q$-systems of semidiscrete type.

Corollary 6.5. Let $(M, \Lambda)$ be an irreducible semidiscrete $Q$-system such that $M$ is an infinite factor and $\lambda^{2} \cong d \cdot \lambda$. Then the dual $Q$-system $(\tilde{M}, \tilde{\Lambda})$ is the crossed product of $(M, \Lambda)$ by an outer action of a discrete Woronowicz algebra.

Proof. The irreducibility condition means that $N^{\prime} \wedge M=Z(M)=\mathbb{C}$. The assertion now follows by the above considerations

We cannot give the result involving semicompact $Q$-systems. Even for actions of compact groups on factors we need additional conditions to assure that a crossed product of a factor by a prime action of a compact group is itself a factor (see [28: Section 21] and [23: Section IV.3]). This problem seems to be directly related to the fact that the irreducibility condition for both semidiscrete and semicompact $Q$-systems is equivalent to the irreducibility of the same kind of inclusion in Jones tower, namely the one with the conditional expectation (see (5.2)), hence we cannot apply the result by Combes and Delaroche [1] to prove the dual statement in Theorem 5.6.

As we said previously, we now show that property (iii) in Proposition 6.4 characterises when a depth 2 factor-subfactor inclusion arises as the crossed product by a Kac algebra.

Theorem 6.6. Let $N \subset M$ be an irreducible inclusion of infinite factors.

(a) The following statements are equivalent:

(i) $M$ is the crossed product of $N$ by an outer action of a compact Kac algebra.

(ii) There exists a sequence of finite index irreducible sectors $\left\{\rho_{i}\right\}_{i \in l} \subset \operatorname{Sect}(M)$ which is a basis for the *-semiring generated by the $\left\{\rho_{i}\right\}$ themselves, such that $\gamma=$ $\oplus_{i} d\left(\rho_{i}\right) \rho_{i}$.

(b) The following statements are equivalent:

(i) $M$ is the crossed product of $N$ by an outer action of a discrete Kac algebra.

(ii) There exists a sequence of finite index irreducible sectors $\left\{\rho_{i}\right\}_{i \in I} \subset \operatorname{Sect}(N)$ which is a basis for the *-semiring generated by the $\left\{\rho_{i}\right\}$ themselves, such that $\left.\gamma\right|_{N}=$ $\oplus_{i} d\left(\rho_{i}\right) \rho_{i}$.

Proof. We only prove part (a), part (b) being analogous.

(i) $\Rightarrow$ (ii): Assume $M$ is the crossed product of $N$ by an outer action of a compact Kac algebra, which is isomorphic to $\gamma(N)^{\prime} \wedge N$ [3]. Then $\gamma(M)^{\prime} \wedge M$ is a discrete Kac algebra isomorphic to $\oplus_{i} \mathcal{B}\left(\mathcal{H}_{i}\right)$, and $d_{i}:=\operatorname{dim}\left(\mathcal{H}_{i}\right)<\infty$ [4]. Finally, if $E \in E(N, \gamma(M))$ is given by $E:=\gamma\left(v^{*} \cdot v\right)$, and $E_{1}:=j_{N} \circ E^{-1} \circ j_{N} \in P(M, N)$ with $j_{N}:=\operatorname{ad} J_{N}$ is the dual weight, it follows from [4] that $\left.E_{1}\right|_{\gamma(M)^{\prime} \wedge M}=\sum_{i} d_{i} \operatorname{Tr}_{i}$, where $\operatorname{Tr}_{i}$ is the canonical (unnormalized) trace on $\mathcal{B}\left(\mathcal{H}_{i}\right)$. Set $T:=E \circ E_{1} \in P(M, \gamma(M))$, let $p_{i}$ be the minimal central projections in $\gamma(M)^{\prime} \wedge M$, and $v_{i} \in M$ be isometries such that $p_{i}=v_{i} v_{i}^{*}$, so that, with $\sigma_{i}:=v_{i}^{*} \gamma(\cdot) v_{i} \in \operatorname{End}(M)$, we have $\gamma \cong \oplus \sigma_{i}$ and $\sigma_{i}(M)^{\prime} \wedge M \cong \mathcal{B}\left(\mathcal{H}_{i}\right)$. Then, by [7: Theorem 6.6 and Corollary 6.10], $p_{i} \in\left(\gamma(M)^{\prime} \wedge M\right)_{T} \cap \mathfrak{M}_{T}$, so that, by the following Lemma 6.7, we have

$$
\operatorname{Ind}\left(T_{p_{i}}\right)=\left(T_{p_{i}}\right)^{-1}\left(p_{i}\right)=T^{-1}\left(T\left(p_{i}\right) p_{i}\right) p_{i}
$$


As $T\left(p_{i}\right)=d_{i}^{2}$ and $j_{M} \circ T^{-1} \circ j_{M}=\gamma^{-1} \circ T \circ \gamma$, so that $T^{-1}\left(p_{i}\right)=d_{i}^{2}$, we get $\operatorname{Ind}\left(\sigma_{i}\right)=$ $\operatorname{Ind}\left(T_{p_{i}}\right)=d_{i}^{4}$. Decomposing each $\sigma_{i}$ in irreducible equivalent sectors $\sigma_{i} \cong d_{i} \rho_{i}$, we get $d_{i}^{2}=d\left(\sigma_{i}\right)=d_{i} d\left(\rho_{i}\right)$, so that $d\left(\rho_{i}\right)=d_{i}$, and therefore $\gamma \cong \oplus_{i} d\left(\rho_{i}\right) \rho_{i}$.

Finally, as Proposition 6.4/(ii) is truc, we obtain $\oplus_{i j} d_{i} d_{j} \rho_{i} \rho_{j} \cong \gamma^{2} \cong d \gamma \cong \bigoplus d d_{i} \rho_{i}$, that is the irreducibles contained in $\rho_{i} \rho_{j}$ are a subset of $\left\{\rho_{k}\right\}$. Moreover, $\oplus d_{i} \bar{\rho}_{i} \cong \bar{\gamma} \cong$ $\gamma \cong \oplus d_{i} \rho_{i}$, so that $\bar{\rho}_{i} \cong \rho_{j(i)}$ for some $j(i)$. All this shows that $\left\{\rho_{k}\right\}$ is a basis for the *-semiring generated by $\left\{\rho_{k}\right\}$.

(ii) $\Rightarrow$ (i): By Theorem $6.3, M=N \times_{\alpha} \mathfrak{A}$ where $\mathfrak{A}$ is a compact Woronowicz algebra, which appears as $\mathfrak{A}=\gamma(N)^{\prime} \wedge N$. The dual algebra $\hat{\mathfrak{A}}$, which is a discrete Woronowicz algebra, is given by $\gamma(M)^{\prime} \wedge M$ and is isomorphic to $\oplus_{i} \mathcal{B}\left(\mathcal{H}_{i}\right), \operatorname{dim} \mathcal{H}_{i}=d\left(\rho_{i}\right)$. Let $E \in E(N, \gamma(M))$ be the unique expectation and $E_{1} \in P(M, N)$ the dual weight, and consider $T:=E \circ E_{1} \in P(M, N)$. It is enough to prove that $\sigma_{\ell}^{T}=i d$ (see [3]). Let $\left\{p_{i}\right\}$ be the minimal central projections of $\gamma(M)^{\prime} \wedge M$. Then, using [11: Proposition 2.8], we obtain that $\left.E_{1}\right|_{\gamma(M)^{\prime} \wedge M} \in P\left(\gamma(M)^{\prime} \wedge M\right)$ and $p_{i} \in \mathfrak{M}_{E_{1}}$. Therefore, using the following lemma 6.7 we can define $T_{p_{i}} \in E\left(M, \sigma_{i}(M)\right)$ where $\sigma_{i}=d\left(\rho_{i}\right) \rho_{i}$, and obtain Ind $\left(T_{p_{i}}\right)=d\left(\rho_{i}\right)^{4}$, which means that $T_{p_{i}}$ is the minimal expectation. Therefore $\left.T_{p_{i}}\right|_{\sigma_{i}(M)^{\prime} \wedge M}$ is a trace. As $\left.T\right|_{\gamma(M)^{\prime} \wedge M}=\left.\sum_{i} T\left(p_{i}\right) T_{p_{i}}\right|_{\sigma_{i}(M)^{\prime} \wedge M}$, it is a trace. The claim follows from [7: Corollary 6.10]

Lemma 6.7. Let $A \subset B$ be an inclusion of von Neumann algebras, $G \in P(B, A)$, $p \in\left(A^{\prime} \wedge B\right)_{G} \cap \mathfrak{M}_{G}$ a non-zero projection in $B$, and set $G_{p}:=G(x) G(p)^{-1} p \quad(x \in$ $\left.\left(B_{p}\right)_{+}\right)$. Then $G_{p} \in E\left(B_{p}, A_{p}\right)$ and $\left(G_{p}\right)^{-1}(x)=G^{-1}(G(p) x) p \quad\left(x \in\left(A_{p}^{\prime}\right)_{+}\right)$.

Proof. The proof is the same as [10: Proposition 1.4], with obvious modifications

We remark that Corollary 6.5 can be stated also in the case of Kac algebras, with obvious minor modification.

\section{Appendix}

Although we have already given a characterisation of the conjugate endomorphism (in the semidiscrete and semicompact cases) in [5], we give here a different one based on the methods of this paper.

Proposition 7.1. Let $M$ be a properly infinite von Neumann algebra and $\rho, \sigma \in$ $\operatorname{End}(M)$. Then the following statements are equivalent:

(i) $E(M, \rho(M)) \neq \emptyset$ and $\sigma=\bar{\rho}$.

(ii) $\sigma \rho \succ i d$ and there is an isometry $v \in(i d, \sigma \rho)$ such that $x v=0$ for $x \in M$ implies $x=0$.

(iii) $\sigma \rho \succ i d$, with $v \in(i d, \sigma \rho)$ an isometry such that $s_{\sigma(M)^{\prime} \wedge M}\left(v v^{*}\right)=1$ and $s_{\sigma \rho(M)^{\prime} \wedge \sigma(M)}\left(v v^{*}\right)=1$.

Proof. (i) $\Longleftrightarrow$ (iii) is [5: Theorem 3.4].

(i) $\Rightarrow$ (ii): Let $E \in E(M, \rho(M))$, consider the inclusion $\bar{\rho} \rho(M) \subset \bar{\rho}(M)$ and set $F:=\bar{\rho} \circ E \circ \bar{\rho}^{-1} \in E(\bar{\rho}(M), \bar{\rho} \rho(M))$. From Proposition 2.3 it follows that there are an isometry $V \in \bar{\rho} \rho(M)$ and a choice of a canonical endomorphism $\gamma^{\prime}$ for the inclusion $\bar{\rho} \rho(M) \subset \bar{\rho}(M)$ such that $\gamma^{\prime}(x) V=V x \quad(x \in \bar{\rho} \rho(M))$ and $V^{*} \gamma^{\prime}(\cdot) V=F$. 
Set $V:=\bar{\rho} \rho(v) \quad(v \in M)$. Then $\bar{\rho} \rho(x) v=v x \quad(x \in M)$. Indeed, as $\gamma^{\prime}=\left.a d \Gamma\right|_{\bar{\rho}(M)}=$ $\left.\bar{\rho} \rho\right|_{\bar{\rho}(M)}[16:$ Proposition 2.4] we get, for all $x \in M$,

$$
\bar{\rho} \rho(\bar{\rho} \rho(x) v)=\bar{\rho} \rho(\bar{\rho} \rho(x)) V=V \bar{\rho} \rho(x)=\bar{\rho} \rho(v x)
$$

and the claim follows from the injectivity of $\bar{\rho} \rho$.

Besides $E(x)=\rho\left(v^{*} \bar{\rho}(x) v\right)(x \in M)$. Indeed,

$$
\bar{\rho} \rho\left(v^{*} \bar{\rho}(x) v\right)=V \bar{\rho} \rho(\bar{\rho}(x)) V=F(\bar{\rho}(x))=\bar{\rho}(E(x))
$$

and the claim follows from the injectivity of $\bar{\rho}$.

Therefore, from Proposition 2.3 it follows that $\Gamma^{*} V V^{*} \Gamma=\Gamma^{*} \bar{\rho} \rho\left(v v^{*}\right) \Gamma=v v^{*}$ is Jones' projection for the inclusion $\bar{\rho} \rho(M) \subset \bar{\rho}(M)$. Hence, if $x \in M$ is such that $x v=0$, then $x v v^{*}=0$ so that $x=0$, as $v v^{*}$ is separating for $M$.

(ii) $\Rightarrow$ (i): Let us set $F(x):=\sigma \rho\left(v^{*} x v\right) \quad(x \in \sigma(M))$. Then $F \in E(\sigma(M), \sigma \rho(M))$ as, if $x \in \sigma(M)$ is such that $F\left(x^{*} x\right)=0$, then $\sigma \rho\left(v^{*} x^{*} x v\right)=0$, that is $x v=0$ so that $x=0$.

Setting now $f:=v v^{*}$ and $L:=\langle\sigma(M), f\rangle$, we want to show that $\sigma \rho(M) \subset \sigma(M) \subset L$ is Jones' basic construction for the inclusion $\sigma \rho(M) \subset \sigma(M)$, by using Proposition 2.2. So we must prove $s_{\sigma \rho(M)}(f)=1$, which follows from [5: Lemma 3.2] and $s_{Z(L)}(f)=1$. But we have $s_{Z(L)}(f) \geq s_{\sigma(M) \wedge} \wedge(f)=1$ as follows from [5: Lemma 3.1]. Therefore, by Proposition 2.5, $\langle\sigma(M), f\rangle=M$.

Finally, to show that $\sigma$ is conjugate to $\rho$, set $\lambda:=\sigma \rho$ and observe that Proposition 2.4 applied to $\lambda$ gives that $\lambda$ is a canonical endomorphism for $N \subset M$, hence $\rho$ and $\sigma$ are conjugate

\section{References}

(1) Combes, F. and C. Delaroche: Groupe modulaire d' une espérance conditionnelle dans une algèbre de von Neumann. Bull. Soc. Math. France 103 (1975), 385 - 426.

(2) Enock, M. and R. Nest: Irreducible inclusion of factors, multiplicative unitaries and Kac algebras. J. Funct. Anal. 137 (1996), $466-543$.

[3] Enock, M.: Inclusions irreductibles des facteurs et unilaires multiplicatifs. Part II. J. Funct. Anal. 154 (1998), $67-109$.

[4] Enock, M. and J. M. Schwartz: Kac Algebras and Duality of Locally Compact Groups. New York: Springer 1992.

[5] Fidaleo, F. and T. Isola: On the conjugate endomorphism in the infinite index case. Math. Scand. 77 (1995), $289-300$.

[6] Fidaleo, F. and T. Isola: Minimal expectations for inclusions with atomic centres. Intern. J. Math. 7 (1996), $307-327$.

[7] Haagerup, U.: Operator valued weights in von Neumann algebras (Part II). J. Funct. Anal. 33 (1979), $339-361$.

[8] Halpern, H., Kaftal, V. and L. Zsido: Finite weighl projections in von Neumann algebras. Pac. J. Math. 147 (1991), $81-121$. 
[9] Hewitt, E. and K. A. Ross: Abstract Harmonic Analysis (Vol. II). New York: Springer 1970.

[10] Hiai, F.: Minimum index for subfactors and entropy. J. Oper. Theory 24 (1990), 301 336.

[11] Izumi, M., Longo, R. and S. Popa: A Galois correspondence for compact groups of automorphisms of von Neumann algebras with a generalization to Kac algebras. Preprint 1996.

[12] Kadison, R. V. and J. R. Ringrose: Fundamentals of the Theory of Operator Algebras (Vol. I and II). New York: Acad. Press 1983 and 1986.

[13] Kosaki, H.: Extension of Jones' theory of index to arbitrary factors. J. Funct. Anal. 66 (1986), 123 - 140.

[14] Kosaki, H.: Characterization of crossed product (properly infinite case). Pac. J. Math. 137 (1989), $159-167$.

[15] Longo, R.: Simple injective subfactors. Adv. Math. 63 (1987), 152 - 171.

[16] Longo, R.: Index of subfactors and statistics of quantum fields (Part I). Commun. Math. Phys. 126 (1989), $217-247$.

[17] Longo, R.: Index of subfactors and statistics of quantum fields (Part II). Commun. Math. Phys. 130 (1990), $285-309$.

[18] Longo, R.: Minimal index and braided subfactors. J. Funct. Anal. 109 (1992), $98-112$.

[19] Longo, R.: A duality for Hopf algebras and for subfactors (Part I). Commun. Math. Phys. 159 (1994), 133 - 150.

[20] Longo, R. and K. H. Rehren: Nets of subfactors. Rev. Math. Phys. 7 (1995), $567-597$.

[21] Mack, G. and V. Schomerus: Quasi Hopf quantum symmetry in quantum theory. Nucl. Phys. B370 (1992), $185 \mathrm{ff}$.

[22] Masuda, T. and Y. Nakagami: A von Neumann algebra framework for the duality of the quantum groups. Publ. Res. Inst. Math. Sci. Kyoto 30 (1994), $799-850$.

[23] Nakagami, Y. and M. Takesaki: Duality for Crossed Products of von Neumann Algebras (Lecture Notes in Mathematics: Vol. 731). New York: Springer 1979.

[24] Nill, F. and H.-W. Wiesbrock: A comment on Jones inclusions with infinite index. Rev. Math. Phys. 7 (1995), 599 - 630.

[25] Paschke, W. L.: Inner product modules over $B^{*}$-algebras. Trans. Amer. Math. Soc. 182 (1973), $443-468$.

[26] Pimsner, M. and S. Popa: Entropy and index for subfactors. Ann. Scient. Ecol. Norm. Sup. (4) 19 (1986), $57-106$.

[27] Popa, S.: Classification of subfactors and of their endomorphisms (Conf. Board Math. Soc. Lect. Notes Ser.: Vol. 86). Providence (Rhode Island): Amer. Math. Soc. 1995.

[28] Stratila, S.: Modular Theory in Operator Algebras. Tunbridge Wells, Kent (England): Abacus Press 1981.


(England): Abacus Press 1979.

[30] Takesaki, M.: Theory of Operator Algebras (Vol. I). New York: Springer 1979.

Received 09.02.1998; in revised form 05.10.1998 